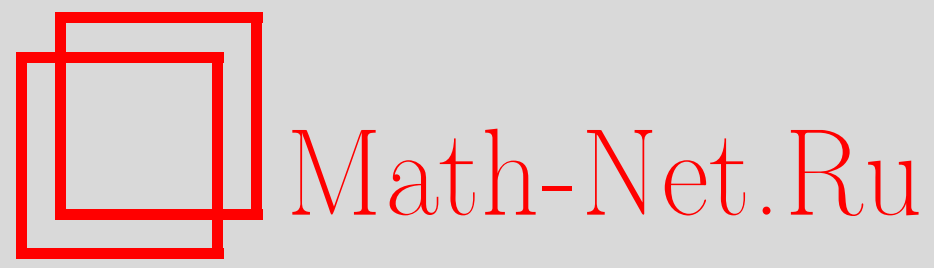

М. А. Бузова, Некоторые свойства ядер уравнений Фредгольма второго рода, Вестн. Сам. гос. техн. ун-та. Сер. Физ.-мат. науки, 2011, выпуск 1(), 28-33

DOI: https://doi.org/10.14498/vsgtu868

Использование Общероссийского математического портала Math-Net.Ru подразумевает, что вы прочитали и согласны с пользовательским соглашением http://www.mathnet.ru/rus/agreement

Параметры загрузки:

IP: 52.6 .47 .48

26 апреля 2023 г., 13:46:28 
УДК 517.9+621.396.6

\section{НЕКОТОРЫЕ СВОЙСТВА ЯДЕР УРАВНЕНИЙ ФРЕДГОЛЬМА ВТОРОГО РОДА}

\section{М. А. Бузова}

Самарское отделение научно-исследовательского института радио, 443011, Самара, ул. Советской Армии, 217.

E-mail: bma@soniir.ru

Выполнены некоторые исследования свойств ядер интегральных уравнений Фредгольма второго рода с особенностями. Рассматриваемые уравнения имеют смысл граничного условия для тангенциальной компоненты магнитного поля $и$ используются в задачах о рассеянии на идеально проводящих рассеивателях конечной толщины. Показано, что ядра таких интегральных уравнений представимы в виде дельта-функиии Дирака. Следствием такой математической формализации является интересный бизический эббект, заключающийся в том, что наведенный ток, найденный по методу физической оптики, численно равен разности токов на освещённой и теневой сторонах рассеивателя, найденных по методу интегральных уравнений.

Ключевые слова: интегральные уравнения, задача рассеяния электромагнитного поля, дельта-функиия Дирака.

Введение. Интегральные уравнения Фредгольма второго рода достаточно широко применяются в различных физических задачах, в том числе и в задачах линейной макроскопической электродинамики [1-3]. Поэтому их исследованиям посвящено большое количество работ. В них освещены как вопросы теоретического характера, так и некоторые практически важные задачи. Однако во многих работах речь идет об исследовании интегральных уравнений с ограниченными вещественными или эрмитовыми ядрами. Рассмотрению же уравнений типа Фредгольма, ядра которых являются не эрмитовыми и имеют особенности, посвящено, на взгляд автора, недостаточное количество работ. В то же время, уравнения с особенностями возникают в некоторых практически важных задачах электродинамики, например, при решении задач рассеяния электромагнитного поля на замкнутых идеально проводящих объектах произвольной формы и размеров. Поэтому представляет интерес более детальное изучение свойств интегральных уравнений, возникающих в физических задачах, с формальной математической точки зрения. Ранее автором уже проводились некоторые исследования в данном направлении $[4,5]$. Данная работа посвящена дальнейшему исследованию свойств ядер интегрального уравнения Фредгольма второго рода, используемых при решении указанных задач.

1. Постановка задачи. Рассмотрим классическую задачу дифракции электромагнитного поля на идеально проводящем рассеивателе. Задача формулируется следующим образом: при заданных сторонних источниках (заданном распределении стороннего поля) необходимо найти полное электромагнитное поле в любой точке пространства. Данная задача относится к классу внешних задач электродинамики, и её решение, как правило, с помощью известных

Мария Александровна Бузова (к.ф.-м.н.), старший научный сотрудник, научный отдел 2. 


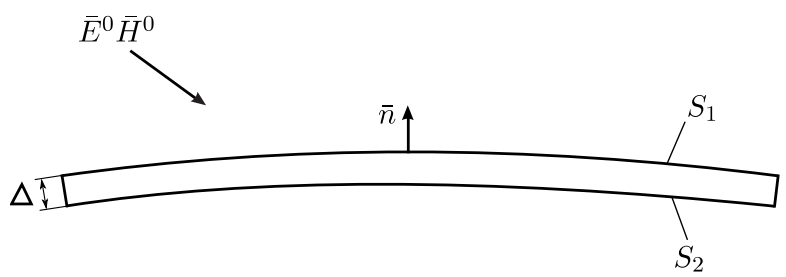

Рис. 1. К постановке задачи

подходов сводится к решению интегральных уравнений относительно наведённых на рассеивателе токов $[1,2]$.

Из всего многообразия рассеивателей для рассмотрения выделим электрически тонкие металлические листы с конечной толщиной $\Delta(\Delta \ll \lambda$, где $\lambda$ - длина волны стороннего поля), электрически большим радиусом кривизны и электрически небольшими поперечными размерами, как показано на рис. 1. Для такого рассеивателя можно пренебречь током на обечайке листа и записать систему интегральных уравнений второго рода для токов на двух сторонах листа: $\vec{j}_{S_{1}}(\vec{r})-$ освещённой и $\vec{j}_{S_{2}}(\vec{r})-$ теневой. Далее приводится первое уравнение системы, соответствующее, к примеру, поверхности листа $S_{1}$ (освещённой), так как второе будет полностью аналогичным:

$$
\begin{aligned}
\vec{n}(\vec{r}) \times \vec{H}^{0}(\vec{r})=\vec{j}_{S_{1}}(\vec{r})+ & \int_{S_{1}} K\left(\vec{r}, \vec{r}^{\prime}\right) \vec{j}_{S_{1}}\left(\vec{r}^{\prime}\right) d s^{\prime}+ \\
& +\int_{S_{2}} K\left(\vec{r}, \vec{r}^{\prime}-\Delta \cdot \vec{n}\left(\vec{r}^{\prime}\right)\right) \vec{j}_{S_{2}}\left(\vec{r}^{\prime}\right) d s^{\prime}, \vec{r}, \quad \vec{r}^{\prime} \in S_{1},
\end{aligned}
$$

где $\vec{n}(\vec{r})$ - внешняя нормаль к поверхности $S_{1} ; \vec{H}^{0}(\vec{r})$ - стороннее магнитное поле; $\vec{j}_{S_{1}}(\vec{r}), \vec{j}_{S_{2}}(\vec{r})$ - поверхностные токи на поверхностях $S_{1}$ и $S_{2}$ соответственно; $K\left(\vec{r}, \vec{r}^{\prime}\right)$ - ядро интегрального уравнения; $\vec{r}, \vec{r}^{\prime}$ - радиус-векторы точки наблюдения и точки источника соответственно; $\Delta$ - толщина рассеивателя.

Ядро интегрального уравнения в данном случае имеет следующий вид [5]:

$$
K\left(\vec{r}, \vec{r}^{\prime}\right)=\frac{1}{4 \pi}\left(\frac{1}{\left|\vec{r}-\vec{r}^{\prime}\right|^{2}}+\frac{i k}{\left|\vec{r}-\vec{r}^{\prime}\right|}\right) \cdot \exp \left(-i k\left|\vec{r}-\vec{r}^{\prime}\right|\right) \cdot\left(\vec{n}(\vec{r}) \times\left(\frac{\vec{r}-\vec{r}^{\prime}}{\left|\vec{r}-\vec{r}^{\prime}\right|}\right)\right),
$$

где $k$ - волновое число.

2. Получение основных соотношений. Рассмотрим сначала первый интеграл в уравнении (1), ядро которого имеет особенность. В [5] показано, что данный интеграл может быть вычислен аналитически. Для этого используется известный приём, а именно: особая точка окружается площадкой малого радиуса, интеграл вычисляется в точке на некоторой малой высоте над площадкой, а затем выполняется предельный переход к нулю по высоте и радиусу площадки. В результате было получено следующее выражение для первого интеграла в (1):

$$
\int_{S_{1}} K\left(\vec{r}, \vec{r}^{\prime}\right) \vec{j}_{S_{1}}\left(\vec{r}^{\prime}\right) d s^{\prime} \approx-\frac{1}{2} \vec{j}_{S_{1}}(\vec{r}) .
$$


Несложно показать, что соотношение (3) будет тем точнее, чем больше радиус кривизны рассеивателя. При бесконечном радиусе кривизны, т. е. для плоского листа, (3) перейдет в точное равенство [5].

Как следует из (3), данное выражение полностью совпадает с определением дельта-функции Дирака. Следовательно, для ядра интегрального уравнения Фредгольма второго рода в данном случае можно записать следующее выражение:

$$
K\left(\vec{r}, \vec{r}^{\prime}\right) \approx-\frac{1}{2} \delta_{S}\left(\vec{r}-\vec{r}^{\prime}\right),
$$

где $\delta_{S}$ - двумерная дельта-функция Дирака.

Рассмотрим теперь свойства ядра второго интеграла в (1). Как следует из явного вида интеграла, при $\Delta \rightarrow 0$, т. е. для бесконечно тонкого листа, данный интеграл полностью совпадает с интегралом в первом слагаемом и, следовательно, его ядро также представимо в виде дельта-функции. При конечной же толщине рассеивателя аналитически вычислить данный интеграл, на взгляд автора, не представляется возможным. Поэтому были проведены численные исследования данного интеграла.

Численные исследования проводились на хорошо апробированной модельной задаче. Рассматривался плоский круглый идеально проводящий диск, возбуждаемый осесимметрично элементарным электрическим вибратором, находящимся на расстоянии $\lambda$ от диска. Благодаря осевой симметрии задачи достаточно рассчитывать распределение тока лишь вдоль радиуса диска, что позволяет существенно сэкономить вычислительные ресурсы.

В частности, проводились сравнения функции $\left|-\vec{j}_{S_{2}}(\vec{r}) / 2\right|$ с модулем образа функции $\vec{j}_{S_{2}}(\vec{r})$ после воздействия на нее соответствующего интегрального оператора при различных значения толщины рассеивателя $\Delta$. На рис. 2 показаны соответствующие результаты. Здесь и далее буквой $A$ обозначен интегральный оператор из (1), а буквой $\xi$ - нормированная к радиусу диска координата, отсчитываемая вдоль него же.

Как видно из представленных данных, для малых $\Delta$ указанная функция

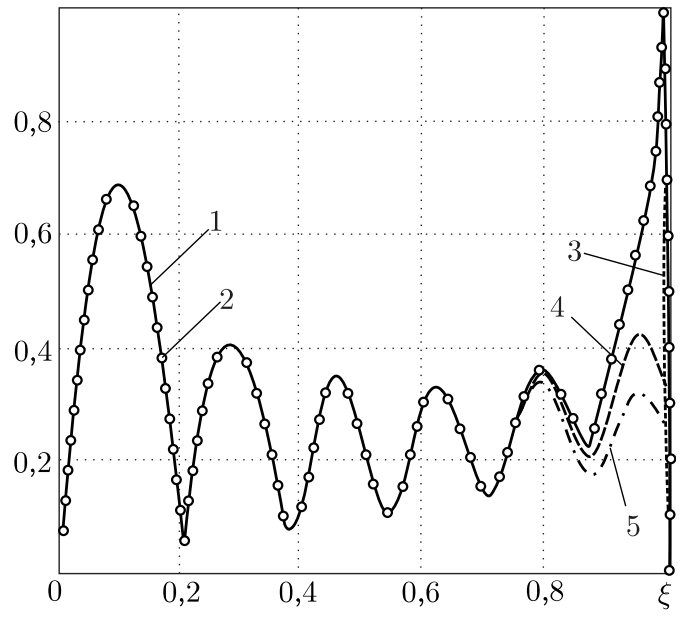

Рис. 2. Графики модуля интегрального оператора, действующего на функцию тока: 1) $\left|-\vec{j}_{S_{2}} / 2\right|$ (сплошная линия); 2) $\left|A \vec{j}_{S_{2}}\right|, \Delta=0,001 \lambda$ (линия с маркерами); 3) $\left|A \vec{j}_{S_{2}}\right|, \Delta=0,01 \lambda$; 4) $\left.\left|A \vec{j}_{S_{2}}\right|, \Delta=0,1 \lambda ; 5\right)\left|A \vec{j}_{S_{2}}\right|, \Delta=0,2 \lambda$ 
и соответствующий образ близки между собой. Только при больших $\Delta$ заметны различия между функцией и ее образом на краю рассеивателя. Отсюда можно сделать вывод, что ядро $K\left(\vec{r}, \vec{r}^{\prime}-\Delta \cdot \vec{n}\left(\vec{r}^{\prime}\right)\right)$ для электрически тонких рассеивателей с высокой степенью точности также представимо в виде дельта-функции.

Таким образом, используя полученные свойства ядер интегрального уравнения Фредгольма второго рода, несложно показать, что уравнение (1) для рассеивателей с электрически малой толщиной вырождается в алгебраическое равенство:

$$
2 \cdot \vec{n}(\vec{r}) \times \vec{H}^{0}(\vec{r}) \approx \vec{j}_{S_{1}}(\vec{r})-\vec{j}_{S_{2}}(\vec{r}) .
$$

Аналогичным образом можно показать, что интегральное уравнение для второй (теневой) поверхности листа $S_{2}$ вырождается в такое же алгебраическое равенство. Поэтому система интегральных уравнений для рассеивателя в целом становится вырожденной. Следовательно, токи $\vec{j}_{S_{1}}(\vec{r})$ и $\vec{j}_{S_{2}}(\vec{r})$ из этой системы определить невозможно; можно найти лишь их взаимосвязь.

3. Результаты исследований. Рассмотрим далее, к каким результатам и выводам приводит выражение (5). Вернёмся к физическому содержанию рассматриваемого вопроса. Как известно, выражение, стоящее слева в (5), есть не что иное, как наведённый ток в рамках метода физической оптики. Следовательно, (5) можно переписать в следующем виде:

$$
\vec{j}_{S}(\vec{r}) \approx \vec{j}_{S_{1}}(\vec{r})-\vec{j}_{S_{2}}(\vec{r}) .
$$

Из (6) можно сделать следующий нетривиальный вывод: с учётом рассмотренных выше допущений ток физической оптики равен разности токов на освещённой и теневой сторонах рассеивателя.

Данный вывод был проверен численным путём при различных $\Delta$ для упомянутой выше модельной задачи. В качестве примера на рис. 3 представлены графики распределения тока физической оптики и разности токов $\vec{j}_{S_{1}}(\vec{r})$ и $\vec{j}_{S_{2}}(\vec{r})$ для толщины диска $0,1 \lambda$. Видно, что данные графики хорошо совпа-

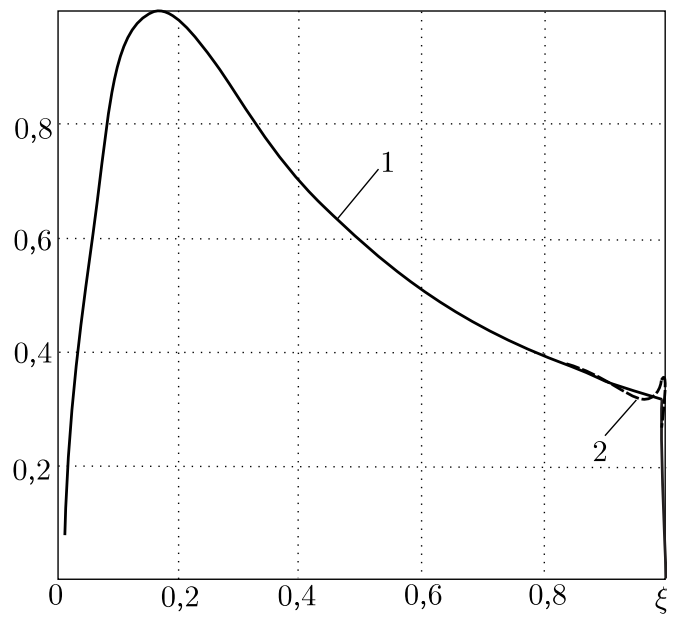

Рис. 3. Графики распределения токов: 1) $\left|\vec{j}_{S}\right|$ (сплошная линия); 2) $\left|\vec{j}_{S_{1}}-\vec{j}_{S_{2}}\right|, \Delta=0,1 \lambda$ (штриховая линия) 
дают между собой. Небольшое различие наблюдается лишь на краю диска, что полностью подтверждает правильность полученных соотношений.

Рассмотрим далее вопросы оценивания погрешности предлагаемого подхода. Как уже было отмечено выше, при большой толщине рассеивателя ядро во втором интеграле уравнения будет отличаться от дельта-функции. Эту поправку можно записать следующим образом:

$$
\kappa=\left|\frac{1}{2} \vec{j}_{S_{2}}(\vec{r})-A \vec{j}_{S_{2}}(\vec{r})\right| .
$$

С другой стороны, эта же поправка равна разности левых и правых частей в (6). Отсюда получается следующее выражение:

$$
\kappa=\left|\vec{j}_{S}(\vec{r})-\left(\vec{j}_{S_{1}}(\vec{r})-\vec{j}_{S_{2}}(\vec{r})\right)\right| .
$$

Эквивалентность выражений (7), (8) проверялась численным путём также для задачи с диском. Расчёты, полученные с помощью формул (7), (8), хорошо согласуются между собой на всём диске (см. рис. 4), что подтверждает правильность проводимых рассуждений. Кроме того, погрешность везде является малой величиной, за исключением края диска. По-видимому, это связано с наличием области возмущенного поля возле кромки диска.

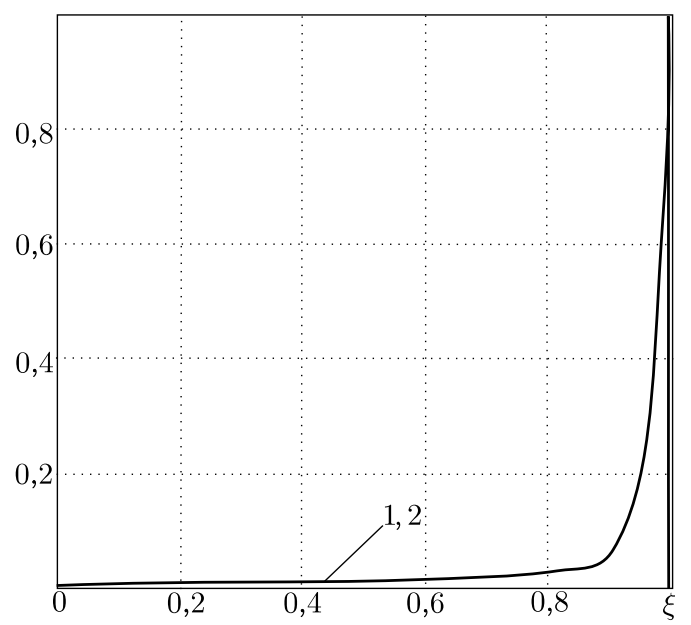

Рис. 4. Погрешность расчётов, вычисленная по (7), (8):

1) $\left.\left|\vec{j}_{S}-\left(\vec{j}_{S_{1}}-\vec{j}_{S_{2}}\right)\right| ; 2\right)\left|\vec{j}_{S_{2}} / 2-A \vec{j}_{S_{2}}\right|, \Delta=0,1 \lambda$

Заключение. В ходе проведенных исследований было показано, что ядро интегрального уравнения Фредгольма второго рода для электрически тонкого рассеивателя с электрически большим радиусом кривизны может быть представлено в виде дельта-функции. Следствием такой математической формализации является интересный эффект, заключающийся в том, что наведённый ток, найденный по методу физической оптики, численно равен разности точных решений: токов на освещённой и теневой сторонах, найденных по методу интегральных уравнений. Этот результат позволяет уточнить наши 
представления о приближенном характере метода физической оптики: данный метод не просто даёт приближенное решение, он даёт «точное» решение, но для разности токов.

\section{БИБЛИОГРАФИЧЕСКИЙ СПИСОК}

1. Васильев E. Н. Возбуждение тел вращения. М.: Радио и связь, 1987. 272 с. [Vasil'ev E. N. Excitation of Bodies of Rotation. Moscow: Radio i Svyaz', 1987. 272 pp.]

2. Computer Techniques for Electromagnetics / International series of monographs in electrical engineering. Vol. 7 / ed. R. Mittra. Oxford - New York - Toronto - Sydney - Braunschweig: Pergamon Press, 1973. 403 pp.; русск. пер.: Вычислительные методы в электродинамике / ред. Р. Миттра. М.: Мир, 1977. 487 с.

3. Купрадзе В.Д. Граничные задачи теории колебаний и интегральные уравнения. М.Л.: Гостехиздат, 1951. 280 c. [Kupradze V.D. Boundary Value Problems in the Theory of Oscillations and Integral Equations. Moscow - Leningrad: Gostekhizdat, 1951. 280 pp.]

4. Бузова М. А. Основные принципы комбинирования методов физической оптики и интегральных уравнений при электродинамическом анализе электрически протяжённых поверхностных рассеивателей // Вестник Самарского отраслевого научно-исследовательского института радио, 2007. № 3(17). C. 44-50. [Buzova M. A. Main principles to combine the physical optics methods and integral equations for electrodynamic analysis of electrically extended surface diffusers // Vestnik Samarskogo otraslevogo nauchno-issledovatel'skogo instituta radio, 2007. no. 3(17). Pp. 44-50].

5. Бузова M.А. Интегральное уравнение второго рода и приближение Гюйгенса-Кирхгоффа в задачах о рассеянии электромагнитного поля на протяженных идеально проводящих рассеивателях // Докл. Акад. Наук, 2010. Т. 431, №4. С. 471-474; Buzova M. A. Second kind integral equation and Huygens-Kirchhoff approximation in the problems of electromagnetic field scattering on lengthy perfectly conducting scatters // Dokl. Physics, 2010. Vol. 55, no. 4. Pp. 164-167.

Поступила в редакцию 20/XII/2010; в окончательном варианте - 12/II/2011.

\section{MSC: 45B05; 78A45}

\section{SOME FEATURES OF SECOND KIND FREDHOLM EQUATIONS KERNELS}

\section{A. Buzova}

Samara Branch of Radio Research and Development Institute, 217, Sovetskaya Armiya st., Samara 443011, Russia.

E-mail: bma@soniir.ru

Kernels of Fredholm integral equations of the second kind with exceptions are analysed in this article. The equations under consideration have a meaning of magnetic field boundary condition and are used in problems of scattering on scatterers with finite thickness. It is shown that these kernels could be stated in a form of Dirac deltafunctions. This mathematical formalization results in interesting physical effect that induced current calculated via physical optics equals the difference of face and back currents of the scatterer, calculated using method of integral equations.

Key words: integral equations, scattering problem for electromagnetic fields, Dirac delta function.

Original article submitted 20/XII/2010; revision submitted $12 / \mathrm{II} / 2011$.

Mariya A. Buzova (Ph. D. (Phys. \& Math.)), Senior Researcher, Research Division 2. 